\title{
Réflexions sur la forme du discours linguistique
}

\author{
Franck Neveu \\ Université de Caen \\ Laboratoire CRISCO (EA 4255)
}

\section{Introduction}

On abordera dans cette contribution à la table-ronde de la thématique «Histoire, Epistémologie, Réflexivité » la question de la forme du discours et de la nécessité de son étude, en limitant toutefois la problématique à un présupposé qui a influé sur la représentation de la science linguistique à l'époque contemporaine, celui d'une science déterminée par sa logique interne et caractérisée, paradoxalement, par une objectivité faiblement médiatisée par la forme du discours.

\section{Sur la forme linguistique du discours scientifique}

La question de la forme du discours dans l'activité scientifique a été débattue assez tardivement, contrairement à ce que l'histoire de la littérature et du sentiment littéraire en Europe fait apparaître relativement à la problématique des formes textuelles.

La philosophie constitue sans doute un excellent observatoire de la question. Denis Thouard (2007) a rappelé récemment comment, à partir des Lumières, particulièrement à partir de l'œuvre de Kant, la philosophie développe une attention accrue à la forme qu'elle se donne pour énoncer ses concepts, attention liée à l'impératif de publicité qui l'anime dans la seconde moitié du XVIII ${ }^{\mathrm{e}}$ siècle, dans un contexte de conscience grandissante des diversités culturelles, sociales et linguistiques.

Le contenu rationnel (pensé comme émancipateur) de la philosophie doit être accessible à tous, mais l'hétérogénéité des publics fait obstacle à la diffusion des concepts et à leur interprétabilité. Le philosophe des Lumières a cessé d'être un producteur d'énigmes conceptuelles mettant au défi l'intelligence, et suscitant une activité herméneutique infinie et égocentrée, pour promouvoir au contraire le partage des idées par le biais d'une exposition claire de ses concepts.

[la philosophie moderne] naît dans le cadre de l'interrogation sur la possibilité d'un système philosophique fondé en raison et susceptible de rendre compte de son fondement. Or une telle ambition ne prend véritablement son élan qu'à partir de la philosophie kantienne pour au moins une raison essentielle : celle-ci s'impose de prendre en compte la subjectivité dans la genèse du système lui-même. Elle ne considère plus celui-ci comme immédiatement donné dans l'entendement. L'esprit ne découvre plus les formes des choses en lui-même ni même hors de lui. Il doit les produire dans une expérience. Il doit indissociablement se connaître et connaître le monde, l'un n'étant pas sans l'autre. La présentation objective du savoir dans un tableau des connaissances ou la reconstitution d'un système de relations dans une caractéristique universelle présentaient toujours un point aveugle qui était l'opérateur intellectuel du système. Le savoir prétendument systématique ne se pensait pas luimême adéquatement et ce déficit de réflexivité ne pouvait plus échapper dès lors que l'on accordait à Kant qu'aucun « donné » ne pouvait comme tel constituer un point de départ pour la philosophie indépendamment des conditions (subjectives) sous lesquelles ce « donné » nous était « donné ». On peut donc considérer que le problème de la philosophie après Kant est bien la question d'un système capable de remonter à ses propres conditions de possibilité, autrement dit capable d'inclure et de légitimer l'instance subjective l'énonçant comme système du savoir ou système de l'absolu. (Thouard, $2007: 8$ ) 
Comme le précise Thouard, Kant a reconnu l'intérêt des concepts de forme et de matière, et en a fait la base de la réflexion, liés qu'ils sont à tout usage de l'entendement :

\begin{abstract}
La forme précède toute matière, la connaissance est en elle-même une mise en forme du divers. [...] La forme met en œuvre l'activité de synthèse du sujet en temporalisant les concepts. Elle leur permet de s'appliquer dans le contexte d'une expérience. La forme en ce sens technique est le « schème ", qui n'est pas l'image ou le schéma, qui sont empiriques, spatiaux, mais le mouvement de formation et de figuration, le principe structurant d'une image. Comme temporalité, le schème introduit une unité différenciée, la règle qui ordonne un divers dans le temps. C'est l'imagination qui produit le schème et permet l'intrusion du concept pur dans le sensible. En restreignant son extension, la schématisation lui confère "sens et signification », la forme déterminant le sensible déterminable. (Ibid.)
\end{abstract}

La question de la forme de la philosophie a ainsi pour enjeu la possibilité d'une philosophie comme science, donc comme système. Cette parenthèse philosophique introduit directement à la question posée par la forme du discours dans l'exposition scientifique, et au rôle qu'y joue la singularité langagière. Comme le note Pierre Bourdieu (2001), un chercheur est " un champ scientifique fait homme », en ce que ses structures cognitives sont homologues de la structure du champ, ce qui a pour conséquence leur nécessaire ajustement aux attentes qui sont inscrites dans le champ. Ce qui définit principalement le champ, en tant que lieu de logiques pratiques, c'est l' " habitus scientifique », caractérisé par une série de routines où s'observe un phénomène d'incorporation de la théorie dans la pratique, c'est, comme le dit Bourdieu (2001 : 83) «"une conscience théorique réalisée", c'est-à-dire incorporée, à l'état pratique ». Pour ce qui concerne la problématique du discours scientifique, l'intérêt de la notion de champ réside dans le fait qu'elle rompt avec le présupposé d'une science pure, c'est-à-dire autonome, mue par la seule puissance de sa logique interne.

La sociologie des sciences corrobore à sa manière les analyses de Thouard sur la forme de la philosophie. Pour accéder au statut de science ou de système la philosophie doit penser sa forme et interroger son mode d'expression conceptuelle, car la science est discours, c'est-à-dire pratiques langagières. Elle se définit notamment, selon Bourdieu, par la possession d'un capital collectif de méthodes et de concepts spécialisés, qui garantissent, explicitement ou non, le «droit d'entrée » dans le champ. Et il convient de noter à cet égard la référence faite à la notion de style, dans cette perspective sociologique, pour caractériser l'habitus disciplinaire comme système de schèmes de perception, d'évaluation et d'expression. Dans cette perspective, le style c'est un ensemble de conditions socio-transcendantales incorporées à la pratique et susceptibles d'exercer un pouvoir de censure :

[J'ouvre ici une parenthèse sur la notion de style : les produits d'un même habitus sont marqués par une unité de style (style de vie, manière, écriture d'un artiste). Dans la tradition de la sociologie de la science, le thème du style est présent chez Mannheim ${ }^{1}$, chez Ludwig Fleck $(1980)^{2}$ qui parle de «styles de pensée », c'est-à-dire d'une «tradition de présupposés partagés » pour une grande part invisibles et jamais mis en question, et aussi de "collectif de pensée », communauté de gens qui échangent régulièrement des pensées : les pensées compatibles avec les présupposés fondamentaux du collectif sont intégrées, les autres rejetées. On a ainsi toute une série d'usages très proches qui valent tantôt pour une discipline dans son ensemble, tantôt pour un groupe, un collectif de pensée qui partage un savoir et des présupposés sur la méthodologie, l'observation, les hypothèses acceptables et les problèmes importants Ian Hacking $(1992)^{3}$ parle aussi de «systèmes fermés de pratique de la recherche » (closed systems of research practice).] Cette notion de « style » est importante pour au moins désigner, montrer du doigt, une propriété des différentes sciences, ou disciplines, qui a été écrasée, obnubilée, dans toute la réflexion sur la science, par le fait que la physique et, plus précisément, la physique quantique a été constituée en modèle exclusif de la scientificité, au nom d'un privilège social converti en privilège épistémologique par les épistémologues et les philosophes, peu armés pour penser les effets d'imposition sociale qui s'exerçaient sur leur pensée. Les frontières de la discipline sont protégées par un droit d'entrée plus ou moins codifié, strict et élevé ; 
plus ou moins marquées, elles sont parfois l'enjeu de luttes avec des disciplines voisines. [...] La notion de champ scientifique est importante parce qu'elle rappelle d'une part qu'il y a un minimum d'unité dans la science, et d'autre part que les différentes disciplines occupent une position dans l'espace (hiérarchisé) des disciplines et que ce qui s'y passe dépend pour une part de cette position. (Bourdieu, 2001 : 129-130)

La forme du discours scientifique (ce qui est appelé «style» en sociologie des sciences), et tout particulièrement la terminologie, jouent donc un rôle crucial dans la constitution des champs ou souschamps disciplinaires, puisqu'ils déterminent et rendent visible l'autonomie du domaine. Cette problématique du droit d'entrée dans le champ, et de sa constitution autonome a été illustrée par ce que Thomas Kuhn $(1962,1977)$ a appelé la «mathématisation ». La "mathématisation » est à l'origine de phénomènes qui visent à renforcer l'autonomie scientifique, et donc à légitimer les activités de la science, et à accroître leur prestige social, notamment en les arc-boutant sur le principe d'objectivité. Ce que la philosophie et la sociologie des sciences ont su faire apparaître, c'est que la « mathématisation » n'est pas seulement une méthodologie, elle est aussi un discours qui énonce, pour reprendre le mot de Bourdieu, un nomos particulier, autrement dit une loi, lieu d'une légalité spécifique, qui repose sur un principe de division, de territorialisation, de construction de la réalité objective irréductible à celui d'une autre discipline, produisant un effet de régulation mais aussi d'exclusion du champ disciplinaire.

Loin de se limiter au domaine des sciences exactes et expérimentales, la «mathématisation » s'exerce également dans les sciences humaines et sociales ${ }^{4}$, et notamment en sciences du langage. Suffisent à en témoigner les nombreux débats portant sur le «noyau dur» de la discipline, ainsi que ceux qui ont été suscités par l'évolution du singulier de Linguistique vers le pluriel de Sciences du langage dans sa dénomination institutionnelle. Comme nous le rappelions il y a peu ${ }^{5}$, la question du «noyau dur » de la science linguistique touche à celle de sa scientificité, et à celle de la place qu'il convient de faire dans son champ à ses domaines connexes. Dans un ouvrage de réflexion épistémologique, Gilbert Lazard (2006 : 17 et sq.) a récemment développé cette problématique à partir des thèses de Gilles Gaston Granger (1987 : 27), qui désignait par le terme de "proto-science » la science linguistique, encore "à l'état naissant », pour ne pas dire immature, eu égard à « la pluralité de ses paradigmes », où « règne [...] une grande hétérogénéité, et même une certaine confusion conceptuelle ». Chez Granger le terme de «protoscience » sert principalement à caractériser les diverses formes de la physique pré-galiléenne, et l'on ne s'étonne pas de retrouver ici l'opposition convenue entre d'une part diversité, hétérogénéité, confusion et d'autre part unité, objectivité, clarté. Ainsi commente Gilbert Lazard :

Il est de fait que les linguistes adeptes des différents « paradigmes » opèrent avec des appareils conceptuels et des terminologies différentes et, s'il leur arrive d'user des mêmes termes, ils ne leur donnent pas la même signification. Aussi l'intercompréhension est-elle au moins difficile. La partition principale est entre les formalistes, pour la plupart adeptes d'une doctrine "générativiste », selon laquelle une "grammaire universelle » est inscrite génétiquement dans le cerveau humain, et les théoriciens plus traditionalistes qu'on groupe sous le nom de «fonctionnalistes », qui pensent que les structures de langues sont fondamentalement conditionnées par leur fonction d'instruments de communication. [...] Cette dispersion est le sort des proto-sciences. À cela s'ajoute bien souvent un certain manque de rigueur dans la définition des concepts utilisés. Beaucoup de termes issus de la tradition grammaticale sont employés couramment dans des conditions telles qu'il convient presque toujours de se demander quel sens leur donnent les auteurs qui les emploient. Rien n'est plus courant que des mots tels que «sujet», «objet direct», "objet indirect», «transitivité », « mode », « aspect», etc. Chacun, bien sûr, évoque dans l'esprit de tout linguiste une certaine représentation en rapport plus ou moins étroit avec les langues qui lui sont le plus familières. Dans le meilleur des cas, ces mots peuvent avoir un sens précis quand ils sont appliqués à la grammaire d'une langue donnée. Mais quelles notions recouvrent-ils en linguistique générale ? Assurément des notions confuses. (Lazard, $2006: 18-19$ )

On peut retenir de cette courte excursion épistémologique que la forme du discours d'une discipline est déterminée à partir d'un principe fondamental de différenciation, et donc d'identité. Elle a ainsi 
nécessairement une fonction de visibilité et d'affichage, qui est exploitée pour souligner la valeur différentielle de son capital, c'est-à-dire pour en permettre la distinction dans un contexte d'indifférenciation présumée. Cet impératif de différenciation explique le retour régulier de l'argument du rasoir d'Ockham, notamment dans les périodes de renouvellement conceptuel et de remembrement des territoires disciplinaires. Pour mémoire, on rappellera le texte de Saussure recadrant un certain positivisme langagier répandu chez les néogrammairiens :

Plus de figures! Ainsi rien que des expressions répondant aux absolues réalités du langage ? [...]

Plus de figures ! C'est un beau programme, qu'on a vite fait de mettre sur le papier. Et que faut-il pour mettre ce précepte en pratique? Peu de chose, simplement n'employer que des expressions répondant aux absolues réalités du langage, classées d'une manière infaillible.

[...] Proscrire la figure, c'est se dire en possession de toutes les vérités, autrement vous êtes radicalement hors d'état de dire où commence et où finit une métaphore.

[...] Ce serait fort beau, si on était un instant persuadé que ceux qui prêtent ce serment ont la moindre idée de ce à quoi ils s'engagent. Plus de figures? Ainsi rien que des termes répondant aux absolues réalités du langage ? Cela équivaut à dire que les absolues réalités du langage n'offrent pas de mystère pour les néo-grammairiens, qu'ils nous les ont dévoilées.

[...] il est tout à fait inutile de se débattre avec la terminologie et de se figurer longtemps avec naïveté que c'est par maladresse ou incomplète disposition du vocabulaire qu'on ne trouve pas les expressions simples. Il n'y a pas du tout d'expression simple pour les choses à distinguer primairement en linguistique; il ne peut pas y en avoir. L'expression simple sera algébrique ou ne sera pas. (Saussure, 2002 : III, II, 13a et 13b, 233-234, 236)

Il semble que sur cette question de l'expression simple en linguistique, régulièrement débattue, selon laquelle une bonne terminologie est possible dès lors qu'elle consiste à équiper un domaine de connaissances d'un vocabulaire qui ne présente pas la variabilité contextuelle et l'instabilité sémantique des unités du langage ordinaire, il soit difficile de dépasser le stade de la pétition de principe ${ }^{7}$. On peut voir dans ce phénomène d'inadéquation entre l'idéal terminologique invoqué et les observables discursifs de la production scientifique une manifestation de la "mathématisation » avancée du domaine, et de sa très faible opérativité. Une explication de ce décalage peut être trouvée dans la confusion usuelle entre terminologie linguistique et métalangue. La terminologie linguistique est un ensemble de métalangues caractérisées par des méthodologies différentes. C'est donc un complexe d'unités, qui ne saurait aucunement offrir des garanties d'économie, de transparence et de cohérence, eu égard à la diversité des activités et des objets de recherche qui constituent la science linguistique. Économie, transparence et cohérence des termes ne sauraient s'obtenir qu'à partir d'un format commun fourni par un sous-champ, seul cadre susceptible d'assurer une stabilité conceptuelle minimale.

Il y a dans cette inadéquation entre l'idéal et la pratique, pour le moins, un paradoxe, lié au fait que, le discours linguistique, dans sa matérialité, a rarement fait l'objet de l'intérêt des linguistes ${ }^{8}$. C'est sans doute que les linguistes se sont longtemps intéressés à la théorie plus qu'à la théorisation, qui relève de la construction du sens, et donc nécessairement également de la construction du discours. Comme le rappelle fort justement Mathieu Valette (2006) :

Un linguiste, en général, aborde la théorie d'un autre comme un système synchronique, voire achronique, en fonction de son objectif scientifique (décrire les faits de langue, expliquer tels phénomènes). Cette conception utilitaire ne nécessite pas qu'il ait une connaissance historique de la théorie. Il s'approprie le modèle et les concepts comme des outils; il doit en connaître la fonction et savoir les manipuler; il lui importe peu d'en connaître l'histoire. À l'inverse, sitôt qu'il s'intéresse à l'invention des concepts, à l'émergence de nouvelles notions, l'historien épistémologue ne peut en occulter la dimension génétique. On attache aujourd'hui, à raison, de plus en plus d'importance aux ratés du discours : autocorrection, lapsus, anacoluthes, etc. Longtemps victimes d'une idéologie de l'homogénéité, ces 


\begin{abstract}
phénomènes apparaissent désormais constitutifs du texte. Ils ne sont plus seulement indices de la construction du sens, mais aussi partenaires de cette construction. De la même façon, nous pensons qu'une théorie est un texte, et que, dans une certaine mesure, elle doit être étudiée dans sa textualité. (Valette, 2006 b : 12)
\end{abstract}

Travailler sur l'observatoire discursif de la science du langage implique de s'interroger tout d'abord sur l'existence de régularités formelles générales susceptibles de caractériser le domaine. C'est ce que l'on appellera ici son idiomaticité.

\title{
3 Sur l'idiomaticité du discours linguistique
}

Comme tout discours scientifique, le discours linguistique est caractérisable par son idiomaticité, c'est-àdire par une forme de préconstruit langagier et culturel que des particularités expressives permettent d'identifier avec plus ou moins de précision. Il s'agit donc là de spécificités propres aux discours de spécialité, comme le rappellent Ghislaine Pesant, Estelle Thibault (1998) :

\begin{abstract}
D'abord, qu'entendons-nous par idiomaticité en langue de spécialité ? Lorsqu'on parle d'idiomaticité on se situe d'emblée dans le système discursif de la langue, qu'il s'agisse de la langue courante ou de la langue de spécialité. En situation de production de texte au premier degré (rédaction en langue maternelle ou dans une langue autre) ou au second degré (traduction ou révision), le langagier doit viser le plus possible à produire un texte qui reflète les habitudes langagières des spécialistes du domaine qu'il traite. La situation discursive si elle déborde la terminologie ne l'exclut nullement; au contraire, le discours la met en oeuvre, la met dans le texte, dans le contexte. L'idiomaticité renvoie ainsi à l'étude des critères de fonctionnement syntaxique du terme dans son réseau de cooccurrents. Pour nous, ces derniers sont des mots qui gravitent autour du terme, alors que les combinaisons plus ou moins étendues, incluant le terme, sont des phraséologismes, ceux-ci pouvant même prendre la forme d'une phrase. [...] Pour accéder au discours de spécialité, il faut d'abord en maîtriser la terminologie, mais surtout connaître certaines particularités de son fonctionnement syntaxique. Ainsi, un texte juridique est idiomatique lorsque les juristes y reconnaissent les particularités expressives propres à leur langue; lorsqu'il reproduit exactement la terminologie du domaine mais surtout les caractéristiques qui font la spécificité de son expression linguistique. Nous éviterons de parler ici de style car, en droit, le style juridique n'est pas unique. Pensons à la langue des lois et règlements, à celle de la procédure, à celle des contrats, etc. Aussi importe-t-il de considérer le domaine d'application en cause. Le style législatif par exemple va jusqu'à comprendre la présentation. Les guides de rédaction législative sont notamment à cet égard très utiles.
\end{abstract}

Ces «empreintes» technolectales, liées à des habitudes langagières propres à une communauté scientifique, se caractérisent notamment par l'emploi de métatermes, c'est-à-dire d'assemblages lexicaux récurrents, régis par un format syntaxique, sémantique, énonciatif et référentiel déterminé. L'idiomaticité, dans les discours de spécialité, repose sur une dialectique particulier/collectif, qui s'observe dans la référence à la notion de spécificité. C'est même là une partie importante du socle définitionnel du terme d'idiomaticité. Une spécificité, c'est-à-dire une singularité qui ne peut être évaluée que dans le cadre d'une généralité (d'une pratique collective) puisqu'elle est censée s'en démarquer. Cette spécificité est toutefois récursive, puisqu'elle est une particularité expressive propre à une communauté de langue (ou de discours) donc à un groupe. On a donc en quelque sorte du collectif dans du collectif. Une autre dimension intéressante de l'idiomaticité est l'altérité langagière. Qu'il s'agisse de séquences figées ou de collocations, on a affaire en effet à des expressions préformées, antérieures dans leur formation à l'énonciation qui les actualise. C'est en quelque sorte la trace d'une pratique collective qui échappe à l'énonciateur. On est sans doute fondé à y voir une réalisation du préconstruit linguistique, au sens que Culioli a donné à ce terme.

L'intérêt de la notion de préconstruit linguistique et culturel est de nous amener à l'essentiel concernant l'idiomaticité, qui est à la fois la représentation conventionnelle du monde, et l'expression 
conventionnelle de cette représentation. Partant de cette approche conventionnaliste de l'idiomaticité, on conçoit la relation qui peut être établie entre idiomaticité et métalangue, puisque dans une perspective terminologique, et quel que soit le domaine, le paramètre conventionnel est bien évidemment crucial. L'unité terminologique est en effet réputée produite par l'attribution conventionnelle d'une étiquette dénominative à une notion. Les définitions terminologiques, en ce qu'elles doivent faire l'objet d'un consensus des experts, sont nécessairement des définitions conventionnelles.

Les critères qui permettent d'établir une distinction entre unités terminologiques et unités lexicales (critères développés notamment par Pierre Lerat, 1995), entrent tous dans le champ d'application de l'idiomaticité.

(i) Un terme appartient à une famille de mots de sens spécialisé dans la langue où il est employé. Il entretient donc des relations de dépendance très étroites au plan de la forme et au plan du contenu avec les autres unités de la série morphologique dans laquelle il s'inscrit.

(ii) Un terme dispose d'une syntagmatique souvent restreinte, qui s'observe notamment dans les co-occurrences conventionnelles qui le caractérisent. Les contraintes sémantiques de sélection des unités terminologiques ont donc fréquemment pour effet de les associer de manière systématique à d'autres unités relevant du même domaine de connaissances.

(iii) Un terme vise à réunir les conditions maximales de transparence sémantique, et à établir un rapport de référence directe et univoque avec son domaine.

Ce dernier critère nous place au cœur de la problématique. Il est fréquemment présenté, on l'a vu, comme définitoire de l'unité terminologique alors que son application, en sciences humaines, et notamment en sciences du langage, reste plus programmatique qu'effective, en raison du caractère nécessairement relatif des notions en usage dans ces domaines de connaissances, et du flou constitutif des mots qui servent à les énoncer.

Pour mettre en relation plus précisément idiomaticité et métalangue, on peut s'appuyer sur certaines propositions concernant la notion de schéma définitionnel formulées par l'équipe de terminologie et de traduction de Lyon 2 (notamment Thoiron, 1998). Dans cette perspective, que l'on rappelle brièvement, le concept, défini comme l'unité d'un système cognitif, est désigné par un terme fonctionnant, lui, au niveau linguistique, au sein d'une terminologie. Ce concept est descriptible par les traits qui le constituent. De même que le concept est divisible en traits conceptuels, le terme est constitué d'un ou de plusieurs éléments de nomination (ex. dans complément d'objet = deux éléments de nomination). Le schéma définitionnel met en relation au plan linguistique et avec des moyens linguistiques non nécessairement élaborés, des traits constitutifs du concept. Autrement dit, il permet le passage du domaine cognitif stricto sensu, non verbalisé, au domaine linguistique. Le terme n'est donc pas en liaison directe avec le concept. Il y a un élément médian : le schéma définitionnel. On pose ainsi l'existence d'une matrice définitionnelle existant au niveau cognitif, matrice définitionnelle partagée par une communauté linguistique, et qui se manifesterait par le biais des définitions individuelles. La dénomination est obtenue par la sélection de quelques éléments du schéma définitionnel, et la définition terminologique résulte, par reformulation, de cette opération de sélection.

Un tel dispositif intéresse directement la question de l'idiomaticité dans la mesure où il permet de formuler une hypothèse quant à la formation du préconstruit linguistique et culturel. On peut en effet partir de l'idée que le terme sténographie, en les sélectionnant, certains traits constitutifs d'un schéma notionnel constitué d'une matrice définitionnelle intégrée, et que la définition terminologique est une (re)formulation de cette sélection. Dans une perspective terminologique, le lieu de formation de l'idiomaticité pourrait être situé dans ce schéma définitionnel. Cette approche doit toutefois être adaptée à la spécificité de la terminologie linguistique, notamment à sa spécificité référentielle, que caractérisent des objets de langage dont la représentation ne saurait être dissociée de l'expression. On entre là dans le mécanisme tautologique de la métalangue. Un mot, un texte, un discours est ce qu'on appelle un mot, un texte, un discours, contrairement aux objets du monde physique, par exemple, dont on peut avoir une connaissance préalable à la dénomination. 
Quel parti tirer de ce dispositif? Une idée simple, mais qui n'en finit pas de poser problème: les dénominations techniques sont dans la langue, et, à cet égard, elles sont soumises aux mêmes phénomènes d'usage que les dénominations de la langue dite ordinaire. Les travaux sur les «langues de spécialité » (entre autres, Lerat, 1995) ont permis de montrer que la spécialisation des discours et des textes est d'abord une affaire de contenus liés à la représentation des connaissances chez les locuteurs, et donc à leur langue, à leur histoire, à leur culture. Dans cette perspective, il n'est guère pertinent de décrire la terminologie linguistique comme un corps de notions réductible à un stock de termes destinés à désignation de ces notions. Il s'agit bien plutôt d'un ensemble d'expressions servant à dénommer, dans une langue particulière et donc dans un environnement culturel déterminé, des constructions conceptuelles qui relèvent d'un domaine de connaissances spécifique, celui de l'étude des langues et du langage. Un discours spécialisé dans l'étude des langues et du langage ne peut donc être borné à une simple nomenclature. Il est fait de dénominations spécialisées (les termes) employées dans des énoncés, et qui à ce titre mobilisent nécessairement les ressources ordinaires d'une langue donnée (Lerat, 1995).

\section{Quelques observables de la singularité langagière dans le texte linguistique}

Ce détour par la relation qui peut être établie entre idiomaticité et métalangue permet de noter qu'en dépit des apparences les discours de spécialité scientifiques sont loin de constituer un obstacle dirimant à la recherche de traits idiolectaux. Et, bien que traversé par l'expression d'un nomos particulièrement structurant, celui de la mathématisation, le discours linguistique n'en constitue pas moins un lieu favorable à l'épanouissement de la singularité langagière.

On examinera quelques-uns des observables susceptibles d'aider au repérage de ces traits idiolectaux, en précisant d'emblée que l'intérêt d'une étude de ces observables peut résider notamment dans l'apprentissage de la lecture du texte linguistique, qu'il s'agisse de textes exposant les résultats d'une activité de recherche, ou de textes de vulgarisation scientifiquement actualisés, comme les grammaires universitaires.

On rappellera tout d'abord que, relativement au domaine de connaissance dans lequel il s'inscrit, le texte linguistique construit une objectivité médiatisée par le discours, ce dont le lecteur n'a pas nécessairement conscience. Il n'existe, comme on sait, aucun objet brut qui puisse être saisi par la science linguistique. Les objets linguistiques sont par nature des objets flous, qui ne se laissent approcher que par des faisceaux de critères. Ils restent principalement des objets de pensée (des catégories, des abstractions), en ce qu'ils sont pour l'essentiel déduits de la forme langagière (graphique ou sonore) sans être matérialisés par cette forme. Cette spécificité référentielle bien connue du texte linguistique n'a bien sûr aucun effet érosif sur les faits de prise en charge de l'énoncé par l'énonciateur.

Un premier poste d'observation très général des singularités langagières du discours linguistique est fourni par le type de terminologie adopté. On entendra par là la fonction qui lui est dévolue. Relativement au domaine de connaissances qui est le sien la terminologie linguistique peut être tenue pour programmatique ou cumulative. L'approche programmatique assigne au vocabulaire scientifique une fonction que l'on peut dire indicative, qui a pour finalité de territorialiser le domaine par le recours à des termes que l'on peut tenir pour des marqueurs méthodologiques et théoriques explicites. En ce cas, il s'agit pour l'énonciateur de dessiner une sorte de chemin lexical et conceptuel, en appuyant sa démarche sur une objectivité strictement déclarative, caractéristique d'une conception scientifique pensée comme nouvelle. Il s'agit d'une approche ouvertement différentielle, qui vise, en instrumentalisant le vocabulaire, à démarquer et à légitimer le champ ou le sous-champ, en lui assignant une place dans le panorama scientifique. Les règles traditionnelles d'économie, d'univocité, d'adéquation et de non-contradiction des termes peuvent trouver à s'appliquer ici. L'approche cumulative, par distinction avec l'approche programmatique, n'est pas en rupture avec l'existant (qu'il s'agisse d'états passés ou présents). Elle le prend en charge, de manière critique ou non, en s'appuyant sur cette antécédence pour construire son discours, quitte à recourir pour cela à des métalangues hétérogènes dont le rendement peut être toutefois jugé positif. Ce pragmatisme terminologique est celui qui caractérise notamment la plupart des 
descriptions grammaticales scientifiquement actualisées, mais qui ne s'inscrivent pas pour autant dans un projet théorique spécifique. De l'une à l'autre approche terminologique le champ d'investigation linguistique peut varier considérablement, notamment, on l'a vu, en raison de la difficulté qu'il y a à maintenir les exigences d'une métalangue tout à la fois non contradictoire, exhaustive et parcimonieuse sur un champ d'application étendu.

En relation directe avec ces orientations terminologiques générales, le recours à la néologie ou aux termes issus de la langue commune constitue un ensemble d'observables de la singularité langagière des plus pertinents. Une des plus sérieuses difficultés terminologiques du discours grammatical réside comme on le sait dans l'instabilité sémantique des termes (nombreux) issus de la langue commune : adjoint, ajout, apport, changement, complément, détachement, groupe, mot, phrase, proposition, racine, texte, par exemple, sont autant de métatermes, qui connaissent une correspondance dans la langue commune, où ils sont par ailleurs usuels, mais avec des valeurs sémantiques très différentes. Cette polysémie n'est pas sans effet sur leurs (maigres) performances terminologiques, en raison des inévitables interférences sémantiques qui se produisent dans l'exercice du discours (à la production comme à la réception). Ces difficultés ne constituent pas forcément un obstacle à leur emploi. Toutefois, celui-ci requiert des précautions méthodologiques et des compléments explicatifs qui ont une incidence directe sur la forme du discours, notamment par le développement des gloses métadiscursives destinées à stabiliser la définition des métatermes. De telles unités, qui contreviennent à l'idéal monosémique de la terminologie, se prêtent donc difficilement à une resémantisation dans le cadre d'un vocabulaire spécialisé, et la pratique du métadiscours alourdit par ailleurs considérablement l'exposition. La néologie constitue un moyen efficace de contourner le problème, sans pour autant annihiler l'opacité. On pensera bien sûr à l'entreprise grammaticale de Damourette et Pichon, fondée sur l'éviction systématique des termes traditionnels: assiette, discordantiel, forclusif, parlure, strument, tiroir, etc. ont d'ailleurs connu une fortune relative dans le discours grammatical français, contrairement aux innombrables composés d'origine grecque auxiamphirhème, diamphithète, épanaschète, épanathète, etc., jugés pour la plupart inexportables. On peut d'ailleurs s'interroger sur ce défaut d'opérativité : tient-il à la forme rébarbative de ces unités, difficilement mémorisables, et faiblement motivées pour la plupart des lecteurs, ou bien à leur stricte monosémie, qui exige une paraphrase de type périphrastique, puisqu'elles ne connaissent pas d'équivalents simples dans la terminologie traditionnelle ? En fait, la question se pose aussi bien pour des unités comme adjet, chronothèse, cosignifiance, effabilité, hyperlangue, forgées dans des œuvres différentes, et qui ont connu des fortunes diverses. Quel que soit le corpus, la performance d'un néologisme se mesure à trois principaux paramètres: le terme doit être justifié, c'est-à-dire définir effectivement un nouveau périmètre notionnel; le terme doit être minimalement motivé sur le plan morphologique, ce qui assure en partie sa lisibilité, et fournit une condition à sa pérennité ; le terme doit présenter une granularité notionnelle satisfaisante, c'est-à-dire limiter le plus possible les interférences sémantiques et les effets de plurivocité. La singularité langagière, dans le domaine terminologique, s'exprime donc aussi par l'application totale ou partielle de ces paramètres.

On a évoqué plus haut le traitement des figures, et particulièrement celui des tropes dans les conceptions normatives de la terminologie linguistique. L'exemple du discours guillaumien permet, s'il en était besoin, de faire apparaître leur performance explicative, et bien sûr de souligner leur rôle déterminant dans l'expression de la singularité langagière. Dans une étude consacrée à la genèse textuelle des concepts scientifiques dans l'œuvre de Guillaume, Mathieu Valette (2006a: 140 et sq.) suit la construction textuelle du concept de mécanisme :

Guillaume avait l'habitude d'opposer la langue en tant qu'elle est mécanique et déterminée, et l'esprit en tant qu'il est libre et indéterminé. Mécanique dérive du grec mêkhanê, «machine ». Ce qui est mécanique peut s'expliquer au moyen de machines ou par analogie aux machines construites, et l'explication mécanique en est un avatar. Guillaume recourt abondamment à l'explication mécanique et il lui est arrivé de comparer la langue à une machine - en de rares occasions, mais jamais de façon anodine. Quatre fois il compare la langue à une machine; et deux fois il prend soin de modérer la portée de la comparaison. Cette réserve est selon nous antiphrastique : plutôt que d'affaiblir la comparaison, elle en souligne l'intérêt. Dans la première 
comparaison, qui date de 1946, Guillaume oppose l'ordre par lequel les différentes pièces d'une machine entrent en branle et l'ordre qui a présidé à l'assemblage par l'ouvrier de cette machine ; ce, pour expliquer que l'ordre en jeu dans la langue en tant qu'ouvrage construit n'a pas de rapport avec sa genèse historique. Dans la seconde, datant de 1947, il compare la relation du sujet parlant et de la langue au mécanicien qui conduit une machine tout en en ignorant l'architecture, le fonctionnement, et les modalités de sa construction [...].

La condensation des deux textes permet à Valette de dégager une équivalence significative chez Guillaume :

\begin{tabular}{|c|c|}
\hline langue & machine \\
\hline /esprit/ ou /sujet parlant/ & /ouvrier/ ou /mécanicien/ \\
\hline /ordre d'apport/ & /construction/ \\
\hline /ordre de rapport/ & /dispositif intérieur/ \\
\hline
\end{tabular}

Comme l'observe Valette, cette bivalence doit être analysée

[...] comme un élément positif du phénomène de conceptualisation, et non comme l'indice d'une vacuité de la comparaison. [...] Sans entrer dans le détail de l'analyse, disons que cette double comparaison participe à un motif complexe où l'énonciation (le mécanicien) et la cognition (l'ouvrier) sont problématisés de concert, et tendent à se confondre : l'homme pensant (alias le sujet parlant) et la pensée humaine (alias l'esprit) sont, d'un point de vue théorique, une seule et même chose. Cela permet de comprendre l'argument de la corporéité du langage avancé par Guillaume pour critiquer la cybernétique. (ibid.)

En tant que tropes ces comparaisons guillaumiennes présentent une valeur descriptive qui reste discutable sur le plan méthodologique. Mais leur valeur suggestive leur confère un intérêt particulier pour l'étude des singularités langagières du discours linguistique :

L'analyse sémantique de textes scientifiques permet d'observer que les théories ne sont pas seulement faites d'idées, de notions et de concepts logiquement articulées, mais de créativité linguistique et de contraintes rhétoriques fortes. On observe ainsi comment des thèmes conceptuels majeurs, comme l'équivalence entre la pensée humaine et l'homme pensant dans les travaux de Guillaume, sans avoir jamais été explicitement formulés, existent néanmoins sous différentes formes textuelles (palinodies rhétoriques, comparaisons, etc.) en lien avec d'autres thèmes (la machine). (Ibid.)

Le corpus guillaumien, sans nécessairement susciter l'adhésion théorique, a souvent fait l'objet de l'intérêt des historiens de la linguistique et des épistémologues, en raison notamment de l'originalité des thèses développées, mais aussi de l'expressivité non académique du linguiste. On n'en retiendra à titre de nouvelle illustration que ces quelques remarques formulées par Michel Launay (1985) :

Le ton de son discours est [...], à bien des égards, celui d'un prophète pour qui le linguiste, en quelque sorte témoin d'une vérité, n'a pas, est-il souvent répété, à théoriser le langage mais à révéler la théorie qu'il est. [...] Il ne serait pas difficile, en accumulant les citations, de dresser de Guillaume un portrait un peu forcé où seraient soulignés le lyrisme et les accents religieux que l'on retrouve parfois dans la rhétorique de certains disciples. C'est là un aspect qui, sans doute, n'a pas peu contribué à sa marginalisation, mais qui ne saurait tout expliquer. Et suffirait-il à rendre compte de sa mise à l'écart qu'il resterait à déterminer ce qu'il pouvait bien y avoir de si fascinant, dans l'objet même du discours de Guillaume, qui pût lui inspirer de tels accents. Cette passion, quoi qu'il en soit, qui traverse son discours, montrait à l'évidence (et c'était peut-être gênant) comment l'objet de la linguistique est aussi objet de désir. (Launay, $1985: 325-326$ ) 
Du côté de la forme des termes, la singularité langagière du discours linguistique est également perceptible aux options « émique » ou « étique » du linguiste, pour reprendre les étiquettes employées en anthropologie culturelle. L'approche «émique » privilégie l'adéquation maximale de l'analyse à l'objet de la description. Dans ce cas, les énoncés descriptifs du linguiste sont contrôlés par le système de fonctionnalités défini dans le cadre de la langue ou du langage décrits. Comme le note Swiggers (1999), les terminologies étroitement liées à telle ou telle tradition grammaticale (tradition arabe, tradition russe, française, etc.), c'est-à-dire liées à une langue particulière, et déterminées par cette langue relèvent de l'approche «émique ». Cela concerne notamment les termes utilisant les formes langagières de la langue objet pour libeller l'étiquette (ex. formes en -ant, formes en -rais, que-phrase, etc.). On notera qu'avec l'usage de ce type de termes il s'agit moins de s'affranchir d'une terminologie traditionnelle jugée lacunaire ou vaporeuse que de mettre l'accent sur une approche résolument non conceptuelle de la terminologie linguistique, c'est-à-dire non explicitement théorisée. L'approche « étique » privilégie quant à elle une description linguistique usant de termes non déterminés par l'objet de la description, mais qui peuvent se superposer aux cas concrets analysés. Dans cette option, les unités terminologiques sont sélectionnées en fonction de leur validité universelle.

On pourrait corréler à cette distinction méthodologique, l'opposition formelle entre termes simples et termes complexes, également significative des choix discursifs et théoriques du linguiste. Les termes simples, qui ne peuvent être que des bases, sont constitués d'unités comportant un seul trait de nomination (ex. nom, verbe, phrase, etc.). Les termes complexes sont composés de plusieurs éléments de nomination, qu'il s'agisse d'unités polylexicales ou non (ex. terme d'adresse $=2$ éléments de nomination, épilinguistique $=2$ éléments de nomination, métalinguistique $=2$ éléments de nomination, coréférence $=$ 2 éléments de nomination, complément d'objet direct $=3$ éléments de nomination, etc.). En démultipliant les traits de nomination, les termes complexes, notamment les termes polylexicaux qui peuvent alterner avec des termes simples (construction détachée vs détachement, construction disloquée vs dislocation, adjectif vs construction adjectivale), complexifient la dénomination tout en l'inscrivant dans une approche de l'observatoire de langue résolument empiriste. Il ne s'agit pas d'une simple attestation, formellement complexe, d'une réalité linguistique également complexe. L'emploi de ces termes suppose en outre l'affichage d'une épistémologie, c'est-à-dire d'une pensée de la langue et des outils nécessaires à son étude. Les traducteurs de discours linguistiques connaissent la difficulté de ce dispositif terminologique, et la nécessité de la prendre en considération dans l'activité traductionnelle. Dans le passage de la langue source à la langue cible, on en vient ainsi à passer de l'idée d'un technolecte sans origine ni destination à l'idée d'un discours orienté, contextualisé, pris en charge et régi, comme tout discours, par un interdiscours.

Sur le plan morphosémantique, comme l'a montré Swiggers (1999), les termes qui entrent dans la constitution d'une terminologie linguistique sont du point de vue des catégories grammaticales et de leur fonction dans le discours :

- des noms (correspondant à des fonctions constantes) : il s'agit de désignateurs de classes, ou d'unités (selon l'actualisation de la tête nominale le/un, la/une, etc.) : ex. le nom, le verbe, le temps, l'aspect, la phrase, etc. ; il s'agit aussi de désignateurs de catégories de propriétés (ex. le présent, le passé, le futur, etc.), ou d'appellatifs de propriétés (ex. l'imparfait). Les appellatifs de propriétés sont fréquemment de nature prédicative (ex. le plus-que-parfait, le passé simple, le futur antérieur, etc.) ;

- des prédicats (de deux types) :

(i) prédicats « de niveau 1 »: il s'agit de prédicats qui décrivent des états, des propriétés, des relations dont sont susceptibles les classes, unités et catégories précédentes : ex. complément circonstanciel, description définie, univers de croyance, etc.

(ii) prédicats « de niveau $2 »$ : il s'agit de prédicats qui décrivent des opérations linguistiques effectuées sur les classes, les unités et les catégories (ex. complémentation, extraposition, topicalisation, thématisation, etc.). 
- des modalisateurs : il s'agit de termes spécifiant la façon dont un prédicat de niveau 1 ou de niveau 2 est applicable aux fonctions constantes des noms (ex. langue flexionnelle, agglutinante, analytique; article défini, indéfini, contracté ; structure superficielle, profonde, etc.

C'est dans les désignateurs de classes, de large extension, qu'on trouve le plus souvent les termes simples, empruntés pour l'essentiel au latin ou au grec, largement stabilisés par l'usage, inscrit, dans la tradition grammaticale, et intervenant (assez tôt dans l'histoire) dans le domaine que l'on peut qualifier de rhétorico-grammatical.

On observe le fréquent transfert des unités entrant dans de la catégorie des modalisateurs vers la catégorie des prédicats de niveau 1 (prédicats de propriétés, de relations, etc.). Ainsi, il y a des morphèmes flexifs et des morphèmes déflexifs. Les termes flexifs et déflexifs apparaissent ici comme des modalisateurs spécifiant la façon dont le prédicat de niveau 1 (morphème flexif/déflexif) est applicable aux fonctions constantes du nom morphème. La substantivation de ces modalisateurs (un flexif, un déflexif) les recatégorise en prédicats de niveau 1 . Ils sont en quelque sorte autonomisés, et prêts, le cas échéant, à subir un nouveau transfert, au moyen d'une ultime modification morphologique, la nominalisation (déflexivité), passant alors dans la catégorie des prédicats de niveau 2, qui décrivent une opération linguistique.

Contrairement à l'opinion généralement répandue, l'adoption de telle ou telle structure morphosémantique en terminologie est moins le fait d'une contrainte méthodologique que d'une stratégie discursive du linguiste, qui opère des choix dans le cadre d'un très large volant de possibles énonciatifs. Qu'il s'agisse de termes renvoyant à des propriétés postulées non observables, de termes « descriptifs » renvoyant à des opérations linguistiques ou à des propriétés observables de la langue objet, ou encore de termes évaluatifs, le format retenu est celui qui est jugé le plus adéquat aux besoins expressifs de l'énonciateur.

On terminera cette évocation rapide des observables de la singularité langagière dans le texte linguistique par le type de rhétorique exploité par l'auteur, et la manière dont il développe son positionnement épistémique dans le discours, c'est-à-dire la manière dont il y inscrit sa conception de l'auctorialité scientifique. En tant que sujet épistémique, l'auteur marque sa singularité par des termes évaluatifs, axiologiques ou non, qui ont pour fonction de renseigner le lecteur sur sa position, conforme ou en rupture, par rapport à une pratique usuelle. Il ne s'agit donc pas à proprement parler de termes, mais d'expressions, pour certaines métadiscursives, destinées à marquer sa spécificité de manière à la rendre explicite au lecteur: des commentaires comme analyse délicate, problème en apparence insoluble, analyse classique, des marqueurs de point de vue épistémique comme contrairement à, à l'inverse, des connecteurs comme en apparence, vraisemblablement, etc. sont susceptibles de jouer un rôle déterminant dans l'explicitation du positionnement épistémique de l'auteur.

Inversement, celui-ci peut recourir à une stratégie d'implicitation, en recourant par exemple à des idées grammaticales endoxales référées à une autorité constituant la source du savoir jamais mentionnée mais omniprésente. On peut ainsi voir s'élaborer, notamment dans les ouvrages de vulgarisation, des prescriptions normatives cryptées. Ainsi (Neveu et Lauwers, 2007) la référence à la « tradition » dans les descriptions grammaticales universitaires joue-t-elle fréquemment sur le perpétuel renvoi à une source énonciative et historique du savoir qui reste obstinément indéterminée : ce que les grammaires appellent, ce que la tradition considère comme, la terminologie traditionnelle, certains grammairiens, on dit, on parle, d'ordinaire, habituellement, généralement, une longue tradition, etc. sont autant de qualificatifs de distanciation, de caractérisants temporels indistincts, de marques de surénonciation susceptibles de sousentendre la pertinence ou la non-pertinence d'un modèle explicatif qui n'est jamais clairement identifié. Plus particulièrement, le recours au mot tradition dans le discours linguistique est plus souvent un argument qu'un ensemble de données historiques précisément circonscrites. Autant d'empreintes de la singularité langagière du discours, susceptibles de recevoir une analyse en termes de traits idiolectaux. 
On notera pour terminer cette évocation de quelques pistes de réflexion concernant la forme du discours linguistique que l'idiomaticité des discours spécialisés, et notamment les contraintes liées à la "mathématisation » du discours linguistique, ne sont bien sûr pas de nature à remettre en cause ces marques de la singularité langagière. Sur ce point, le discours linguistique souffre toutefois d'un déficit d'analyses, que les études terminographiques et le développement de l'approche génétique des textes scientifiques peuvent heureusement corriger. On insistera pour finir sur l'importance d'un examen des observables de la singularité langagière dans l'apprentissage universitaire de la lecture du texte linguistique, qu'il s'agisse du texte grammatical ou du texte lexicographique. Seul ce type d'approche permet de déconstruire efficacement le présupposé d'une objectivité non médiatisée, et d'une science linguistique uniquement déterminée par sa logique interne.

\section{Références bibliographiques}

Auroux S., 1989, «Le langage et la science : une visée historique », in M.-J. Reichler-Béguelin (dir.), Perspectives méthodologiques et épistémologiques dans les sciences du langage, Berne, Peter Lang : 51-68.

Auroux S., 1998, La raison, le langage et les normes, Paris, PUF.

Bachelard G., 1938/1977, La formation de l'esprit scientifique, Paris, Vrin.

Berrendonner A., 1982, L’éternel grammairien. Étude du discours normatif, Berne, Peter Lang.

Bourdieu P., 2001, Science de la science et réflexivité, Paris, Raisons d'agir.

Colombat B., Savelli M. (dir.), 2001, Métalangage et terminologie linguistique, Leuven, Peeters.

Culioli A., 2002, Variations sur la linguistique, Paris, Klincksieck.

Dahl T., 2004, «Textual metadiscourse in research articles : a marker of national culture or of academic discipline ? », Journal of Pragmatics, $36: 1807-1825$.

Dahl T., 2005, «Cultural identity in academic texts », Akademisk Prosa, 3, Skrifter fra KIAP, Romansk institutt, Universitetet i Bergen : 35-45.

Depecker L. (dir.), 2005, « La terminologie : nature et enjeux », Langages, 157.

Détrie C. et Neveu F. (dir.), 2005, «L’idiolecte. Du singulier dans le langage », Cahiers de Praxématique, 44, Université de Montpellier III.

Ducrot O., 1992, «Entretien » in C. Lopez Alonso \& A. Séré De Olmos, Où en est la linguistique ? Entretiens avec des linguistes, Paris, Didier Érudition.

Granger G. G., 1987, Leçon inaugurale faite le 7 mars 1987, Paris, Collège de France.

Grossmann F., Rinck F., 2004, « La surénonciation comme norme du genre : l'exemple de l'article de recherche et du dictionnaire en linguistique », Langages, $156: 34-50$.

Grunig B.-N., 2002, « Linguistique et brouillons, dynamique et synchronisation », Langages, 147 : 113-123.

Halliday M.A.K., 2004, The language of Science, London, Webster, Continuum.

Jeanneret Y., 1998, L'affaire Sokal ou la querelle des impostures, Paris, PUF.

Jeanneret Y., 2000, «Des médias, des sciences et des textes : régimes actuels de construction des objets et des paroles scientifiques ", in F. Cusin-Berche, Les Carnets du CEDISCOR, 6, Presses Sorbonne Nouvelle : 199-216.

Kuhn T., 1962, The Structure of Scientific Revolutions, Chicago, University of Chicago Press; trad. fr. de L. Meyer, La structure des révolutions scientifiques, Paris, Champs-Flammarion, 1983.

Kuhn T., 1970, « Postface - $1969 »$, in Seconde édition augmentée de Kuhn (1962).

Kuhn T., 1977, The Essential Tension, Chicago, The Chicago University Press; trad. fr. de M. Biezunski, P. Jacob, A. Lyotard-May et G. Voyat, La tension essentielle, Gallimard, 1990.

Launay M., 1985, «Gustave Guillaume : la loi et le symptôme », in S. Auroux et alii, La linguistique fantastique, Paris, Clims, Denoël : 324-338. 
Lazard G., 2006, La quête des invariants interlangues. La linguistique est-elle une science ?, Paris, Champion.

Leduc-Adine J.-P., Vergnaud J. (dir.), 1980, « La terminologie grammaticale », Langue française, 47.

Leeman D. (dir.), 1979, « Sur la grammaire traditionnelle », Langue française, 41.

Lerat P., 1995, Les Langues spécialisées, Paris, PUF.

Milner J.-C., 1989, Introduction à une science du langage, Paris, Éditions du Seuil.

Neveu F., 2000, Lexique des notions linguistiques, Paris, Nathan, coll. «128».

Neveu F., 2004, Dictionnaire des sciences du langage, Paris, Armand Colin, coll. « Dictionnaires ».

Neveu F. (dir.), 2006, Syntaxe \& Sémantique, n 7, «La terminologie linguistique. Problèmes épistémologiques, conceptuels et traductionnels », Presses Universitaires de Caen.

Neveu F., 2007, «Les fondements normatifs de la terminologie linguistique et l'observatoire discursif de la science du langage », in G. Siouffi et A. Steuckardt, Les Linguistes et la norme, Berne, Peter Lang : 123-148.

Neveu F. et Lauwers P. 2007, "La notion de 'tradition grammaticale' et son usage en linguistique française », Langages, $167: 7-26$.

Neveu F. et Pétillon S. (dir.), 2007, Sciences du langage et sciences de l'Homme, Limoges, Lambert-Lucas.

Nuchèze V. de, 1998, « Approche pragmatico-énonciative des discours de recherche », Lidil, $17: 25-40$.

Pesant G. et Thibault E., 1998, « Pour une combinatoire phraséologique de la publicité des droits », Méta, XLIII, 2.

Rinck F., 2005, «Images of scientific activity through the research article : a comparison between linguistics and literary studies », in. K. Fløttum and O. Korsnes, Academisk Prosa, n 3, Department of Romance Studies, University of Bergen : 75-86.

Roe P., 1977, Scientific Text, ELR, University of Birmingham.

Saussure F. de, 2002, Écrits de linguistique générale, Paris, Gallimard, éd. S. Bouquet \& R. Engler.

Sokal A. et Bricmont J., 1997, Impostures intellectuelles, Paris, Odile Jacob.

Stéfanini J., 1994, Histoire de la grammaire, Paris, CNRS Éditions.

Sumpf J., 1972, «Les traits principaux de la tradition linguistique française », Langue française, 14 : 70-98.

Swiggers P., 1999, «Pour une systématique de la terminologie linguistique : considérations historiographiques, méthodologiques et épistémologiques », in A. Lemaréchal (dir.), La Terminologie linguistique, Mémoires de la Société de Linguistique de Paris, nouvelle série, tome VI, Paris, Peeters : 11-49.

Swiggers P., Janse M., 1991, «Les dictionnaires de terminologie linguistique : bibliographie systématique », Meta, XXXVI, $4: 647-653$.

Thouard D., 2007, Le partage des idées. Études sur la forme de la philosophie, Paris, CNRS Éditions.

Thoiron P., 1998 : «Figement, dénomination et définition », in S. Mejri et alii (dir.), Le Figement lexical, Actes de la $1^{\mathrm{e}}$ R.L.M, Tunis, CERES : 219-238.

Touratier C., 1998b, «Les grammaires universitaires françaises de ces dix dernières années », Le français moderne, tome LXVI, $1: 73-102$

Valette M., 2006 a, «La genèse textuelle des concepts scientifiques. Étude sémantique sur l'œuvre du linguiste Gustave Guillaume », Cahiers de lexicologie, $89: 125-142$.

Valette M., 2006 b, Linguistiques énonciatives et cognitives françaises, Paris, Champion.

\footnotetext{
${ }^{1}$ Manheim K. (1956), Idéologie et utopie, Paris, Rivière.
} 
${ }^{2}$ Fleck L. (1980), Entstehung und Entwicklung einer wissenschaftlichen Tatsache, Francfort/Main, Suhrkamp (trad. américaine, Genesis and Development of a Scientific Fact, Chicago, University of Chicago Press.

${ }^{3}$ Hacking I. (1992) «The Self-Vindication of Laboratory Sciences», in A. Pickering, Science as Practice and Culture, Chicago, University of Chicago Press : 29-64.

${ }^{4}$ Une illustration de l'impact sociétal de la «mathématisation» dans la forme du discours scientifique nous est donnée avec ce que l'on a appelé «l'affaire Sokal», qui a inauguré un nouvel épisode de la guerre des sciences, bâti autour du triomphe du texte. "Transgresser les frontières : vers une herméneutique transformative de la gravitation quantique », tel était le titre de l'article parodique publié par Alan Sokal et Jean Bricmont en 1996 dans la revue Social Text, simulacre satirique destiné à dénoncer l'usage intempestif de la terminologie scientifique et son exportation abusive des sciences exactes vers les sciences humaines et sociales (Sokal et Bricmont, 1997). Audelà des postures ou impostures terminologiques présumées, ce qui était visé était principalement le « relativisme de l'objectivité en sciences humaines ", et son statut de pure et simple convention sociale. On trouvera dans Jeanneret (1998) une des meilleures analyses de l'« affaire », et des motivations de ses principaux protagonistes.

${ }^{5}$ Neveu et Pétillon (2007).

${ }^{6}$ On pourrait mettre ce texte en perspective avec le célèbre chapitre de la Formation de l'esprit scientifique de Bachelard, consacré aux obstacles épistémologiques de nature verbale, afin de souligner que les débats autour de la forme langagière du discours scientifique ont le plus souvent tourné autour de la question terminologique, et plus particulièrement autour de la place qui doit être accordée à la métaphore : «Nous voulons [...] être encore plus précis et considérer un cas où une seule image, ou même un seul mot, constitue toute l'explication. Nous prétendons caractériser ainsi, comme obstacles de la pensée scientifique, des habitudes toutes verbales. [...] il s'agira d'une explication verbale par référence à un substantif chargé d'épithètes, substitut d'une substance aux riches puissances. Ici, nous allons prendre le pauvre mot d'éponge et nous allons voir qu'il permet d'exprimer les phénomènes les plus variés. Ces phénomènes, on les exprime : on croit donc les expliquer. On les reconnaît : on croit donc les connaître. Dans les phénomènes désignés par le mot éponge, l'esprit n'est cependant pas la dupe d'une puissance substantielle. La fonction de l'éponge est d'une évidence claire et distincte, à tel point qu'on ne sent pas le besoin de l'expliquer. En expliquant des phénomènes par le mot éponge, on n'aura donc pas l'impression de verser dans un substantialisme obscur; on n'aura pas davantage l'impression qu'on fait des théories puisque cette fonction est tout expérimentale. À l'éponge correspond donc un «denkmittel» de l'empirisme naïf. [...] Le danger des métaphores immédiates pour la formation de l'esprit scientifique, c'est qu'elles ne sont pas toujours des images qui passent; elles poussent à une pensée autonome; elles tendent à se compléter, à s'achever dans le règne de l'image ». (Bachelard, 1938/1977: 73, 81)

${ }^{7}$ Voir sur ce point Lazard (1999) et Swiggers (1999). Voir également Ducrot (1992) et Culioli (2002): «La formalisation est un moyen très radical pour sortir de la langue, pour la critiquer. À ce titre, l'idéal de formalisation reste un idéal extrêmement estimable. Mais, de plus en plus, je crois que c'est un idéal irréalisable dans la mesure où les concepts formels que l'on définit pour parler de la langue, dès qu'on essaie de les définir de manière explicite, on les définit au moyen de mots de la langue » (Ducrot, $1992: 68$ ) ; « Une fois qu'on a dit qu'il n'y a pas de métalangue ultime - et puis tout le monde à ce moment-là ressort le théorème d'incomplétude de Gödel - le linguiste, son travail, ce n'est pas de se tordre les mains en se disant : on ne pourra jamais rien faire. Mais c'est de faire, et d'éclairer ce qui peut être éclairé à un moment donné. Alors attention aussi, parce qu'on a l'impression que l'on est toujours dans des apories, que l'on est toujours en train de finasser, de jouer sur les mots. En fait, non : avec ces histoires de métalangue, il ne faut pas se laisser impressionner par ceux qui vous diraient: dans ce cas, vous ne pouvez rien faire. Si, on peut faire quelque chose » (Culioli, $2002: 108-109)$.

\footnotetext{
${ }^{8}$ Voir néanmoins Berrendonner (1982).
} 